\title{
Anthocyanins and human health: How gastric absorption may influence acute human physiology
}

\author{
Iva Fernandes*, Victor de Freitas and Nuno Mateus \\ Chemistry Investigation Centre (CIQ), Department of Chemistry and Biochemistry, Faculty of Sciences, \\ University of Porto, Porto, Portugal
}

\begin{abstract}
This review aims to highlight some aspects regarding anthocyanins bioavailability. Although there is a considerable variability in the values for the biokinetic parameters, anthocyanins appear to be rapidly absorbed and eliminated, reaching low maximal concentrations in plasma and urine. Nevertheless, some works have reported a high content of intact anthocyanins in plasma, possibly resulting from gastric absorption. So far, no anthocyanin metabolites have been detected in the stomach and the possible mechanism of anthocyanin gastric absorption is still unknown. Some progress in this field is expected to be obtained through studies involving a new human cell culture model of the gastric surface suitable for bioavailability screening of nutraceuticals like anthocyanins and anthocyanin-derived pigments. The positive health effects associated with anthocyanin consumption could derive from the contribution afforded by the stomach to intact anthocyanin absorption. Nonetheless, these latter may be further metabolized to other bioactive forms.
\end{abstract}

Keywords: Anthocyanins, bioavailability, gastric absorption, metabolism, microbiota

\section{Introduction}

Anthocyanins are one of the most widespread families of natural pigments in the plant kingdom. Over the years, the scientific community has been focusing on these amazing molecules trying to understand anthocyanins and their properties. Many different chemical structures from numerous natural sources have been characterized and their physical-chemical properties determined [1]; their benefits for human health are unquestionable, and the applications of anthocyanins as colorants or bioactives have been exploited by food, pharmaceutical and cosmetic industries.

*Corresponding author: Iva Fernandes, Chemistry Investigation Centre (CIQ), Department of Chemistry and Biochemistry, Faculty of Sciences, University of Porto, 4169-007 Porto, Portugal. Tel.: +351 220402562; E-mail: iva.fernandes@fc.up.pt.

\section{Chemical features of anthocyanins}

Anthocyanins are responsible for the unlimited diversity of colours from orange and red through purple and blue hues of several fruits, vegetables and plants. The most commonly known anthocyanins are based on six anthocyanidins: cyanidin, delphinidin, malvidin, pelargonidin, peonidin and petunidin (Fig. 1) but there are almost 600 anthocyanins reported to be isolated from plants [2]. These compounds differ in the hydroxylation and methoxylation patterns of ring B. Anthocyanins can be glycosylated in the 3-OH, 5$\mathrm{OH}$ and less commonly in the 7-OH position and, to a lesser extent, in both positions 3-OH and 5-OH (3,5-Odiglycosides). The sugar moieties vary but are usually a mono or disaccharide unit, frequently glucose, galactose, rhamnose, arabinose, rutinose or xylose [3]. The sugar moiety may also be attached to aliphatic or aromatic acids. 


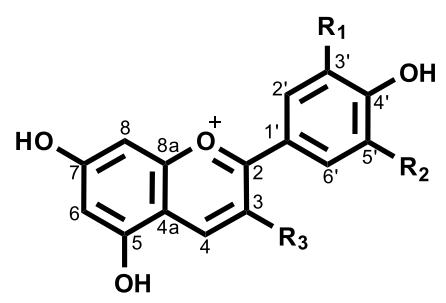

\begin{tabular}{llll}
\hline Anthocyanins & $\mathrm{R}_{1}$ & $\mathrm{R}_{2}$ & $\mathrm{H}$ \\
\hline Pelargonidin $(\mathrm{Pg})$ & $\mathrm{H}$ & $\mathrm{H}$ \\
Peonidin (Pn) & $\mathrm{OCH}_{3}$ & $\mathrm{OH}$ & $\mathrm{H}$ \\
Cyanidin (Cy) & $\mathrm{OCH}_{3}$ & $\mathrm{OCH}_{3}$ & \\
Detunidin (Pt) & $\mathrm{OCH}_{3}$ & $\mathrm{OH}$ & $\mathrm{OH}$
\end{tabular}

Fig. 1. General structure of the six anthocyanidins present in higher plants (flavylium form), $\mathrm{R}_{3}=\mathrm{OH}$. For the anthocyanins glycosylated in the $3-\mathrm{OH}, \mathrm{R}_{3}=$ sugar.

The physical-chemical properties of anthocyanins, particularly their colour characteristics and stability are unique. Anthocyanins are highly reactive molecules and thus sensitive to degradation reactions. Several factors such as oxygen, temperature, light, enzymes and $\mathrm{pH}$, affect anthocyanins chemistry and consequently their stability and colour. The degradation of anthocyanins may occur during their extraction, food processing and storage. Due to their high chemical reactivity, anthocyanins may also be ingested as anthocyanin-derived pigments $[4,5]$. Direct reactions between anthocyanins and flavanols have already been demonstrated in plant extracts and food matrices, especially in red wine where these compounds play an important role in the colour [6-9].

A critical factor that is strictly related to the colour displayed by anthocyanins is the fact that they occur in five species under equilibrium in aqueous solution depending on the $\mathrm{pH}$ : flavylium cation, carbinol base, chalcone, quinonoidal base and anionic quinonoidal base (Fig. 2) [10-13].

The complex chemistry of these compounds is a critical point when considering their absorption in the gastrointestinal (GI) tract. After consumption in their red flavylium cation form, anthocyanins may undergo some transformations in the oral cavity as a result of the more alkaline $\mathrm{pH}$, the physiological temperature, the action of phase II enzymes and the action of the oral microbiota $[14,15]$. The consequent passage through the gastric cavity may account for further changes in the anthocyanin chemical forms. After intestinal absorption, anthocyanins will be mainly in their colourless pseudobase form but may also occur as yellow chalcone and blue quinoidal forms (Fig. 3).

\section{Anthocyanin absorption and metabolism}

The study of flavonoid bioavailability is a quite challenging taste, that becomes even more complex when concerning anthocyanins, as a result of their unique physical-chemistry [16]. According to the reported data some hypothetic pathways of anthocyanins absorption, distribution, metabolism and excretion can be summarized (Fig. 4).

Generally, the scientific community considers that anthocyanin intestinal absorption is around $1 \%$ of the oral ingested amount [17]. However, additional factors may contribute to the proposed low anthocyanin bioavailability such as high rates of cellular uptake, metabolism and excretion [18]. 


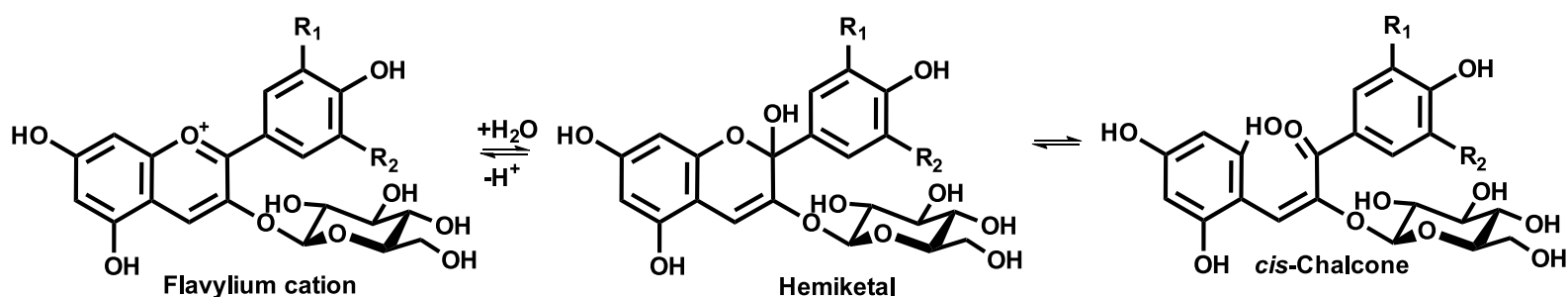

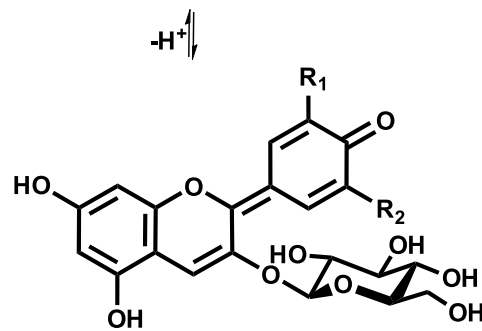

Quinoidal base<smiles>[R]C1=CC(=C2Oc3cc(O)cc(O)c3C=C2O[C@H]2O[C@@H](CO)[C@H](O)[C@H](O)[C@H]2O)C=C([R])C1=O</smiles>

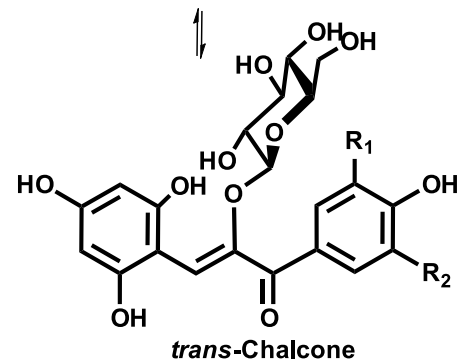

Fig. 2. Anthocyanins equilibrium in aqueous solution depending on $\mathrm{pH}$ [13]. $\mathrm{R}_{1}$ and $\mathrm{R}_{2}=\mathrm{H}, \mathrm{OH}$ or $\mathrm{OCH}_{3}$.

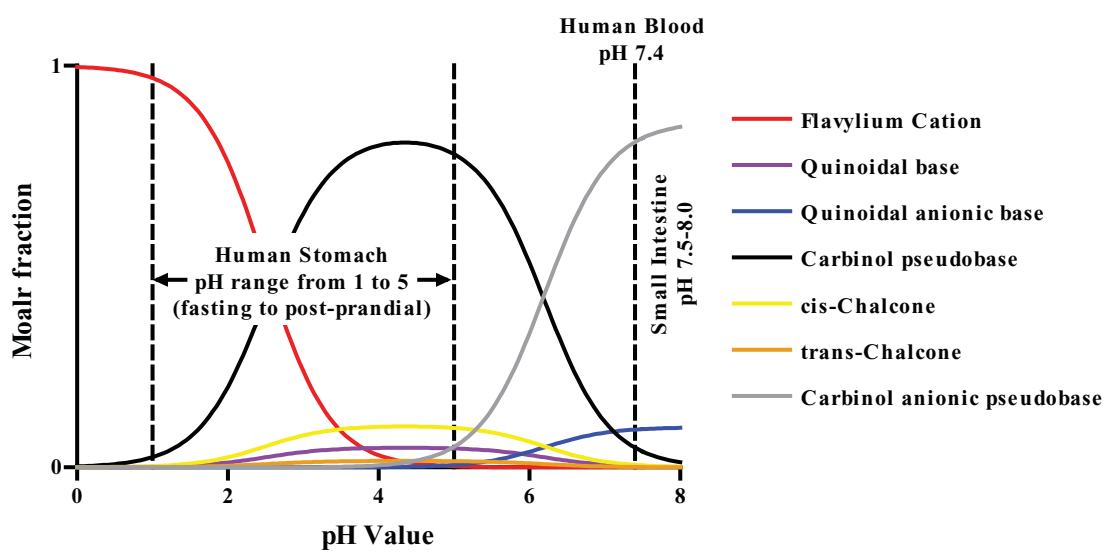

Fig. 3. Schematic representation of the molar fraction of anthocyanin according to the GI tract pH, determined according to [119].

Before reaching the intestinal border several binding events involving anthocyanins may occur, namely interaction with food proteins or with salivary proteins and digestive enzymes [19-21]. A recent work with healthy volunteers has shown that black raspberry anthocyanins could be detected under their hydrolyzed aglycone form in the oral cavity resulting from the activity of $\beta$-glycosidase derived both from bacteria and oral epithelial cells [14]. In the same study, parent anthocyanins and cyanidin-3-glucoside microbiota metabolite, protocatechuic acid, were detected in the saliva. In addition, saliva samples were found to contain glucuronidated anthocyanin conjugates, consistent with intracellular uptake and phase II conversion of anthocyanins [14].

The oral transformations observed in this study have to be analyzed with some caution as a result of the short period of time that these compounds remain in the oral cavity.

After ingestion, anthocyanins are readily detected in plasma in their parent forms, which may suggest their absorption through the gastric wall [22-25].

Although only native anthocyanins have been detected in the stomach [26], the enzymatic machinery (UDP-glucuronosyltransferase, sulfotransferase, and catechol- $O$-methyl transferase) essential for antho- 


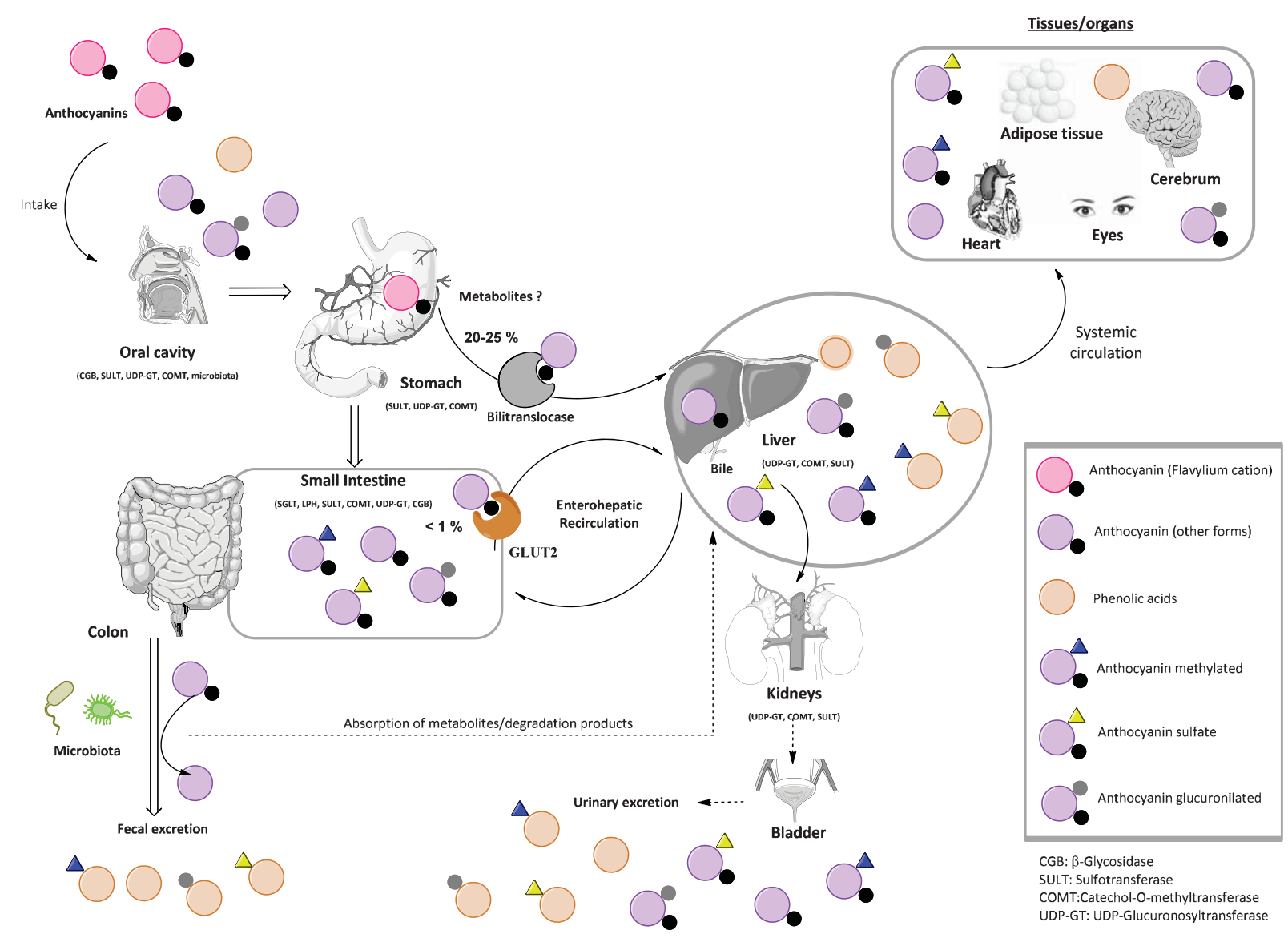

Fig. 4. Hypothetic pathways of anthocyanins absorption, distribution, metabolism and excretion according to the reported data.

cyanin conjugation is present in the gastric mucosa [27-30]. Additionally, some in vitro studies have shown that some flavonoids could be metabolized into glucuronidated and sulphated metabolites by the gastric wall $[31,32]$.

Unabsorbed anthocyanins enter the more basic conditions of the small intestine predominantly in their carbinol pseudobase form (Fig. 3). Unlike other flavonoids glycosides that are hydrolyzed, anthocyanin glycosides are rapidly and efficiently absorbed in the small intestine [33-35]. Anthocyanins may be absorbed into the intestinal epithelial cells through the glucose transporters such as glucose transporter 2 (GLUT2) [36] and possibly $\mathrm{Na}^{+}$/glucose cotransporter 1 (SGLT1) [37]. One recent study indicated that several anthocyanins may be actively transported out of the intestine and endothelia, limiting their bioavailability to the circulation [38]. The efflux of at least some anthocyanins back into the lumen of the small intestine is thought to involve members of the adenosine triphosphate (ATP)-binding cassette (ABC) family of transporters including multidrug resistance protein (MRP) and P-glycoprotein (P-gp) [38].

Furthermore, anthocyanins have also been reported to be quickly metabolized and to appear in the circulation or into bile and urine as both intact and metabolized forms (glucuronidated, sulphated or methylated derivatives) [33, 34, 39-43].

Unabsorbed anthocyanins reach the colon where they undergo substantial structural modifications. Previous studies have suggested that this is likely due to the spontaneous degradation under physiological conditions [44] or following microbial metabolism. In fact, colonic microbiota hydrolyses glycosides into aglycones and degrades them to simple phenolic acids (Fig. 5).

According to Vitaglione and co-workers protocatechuic acid is the major human metabolite of 

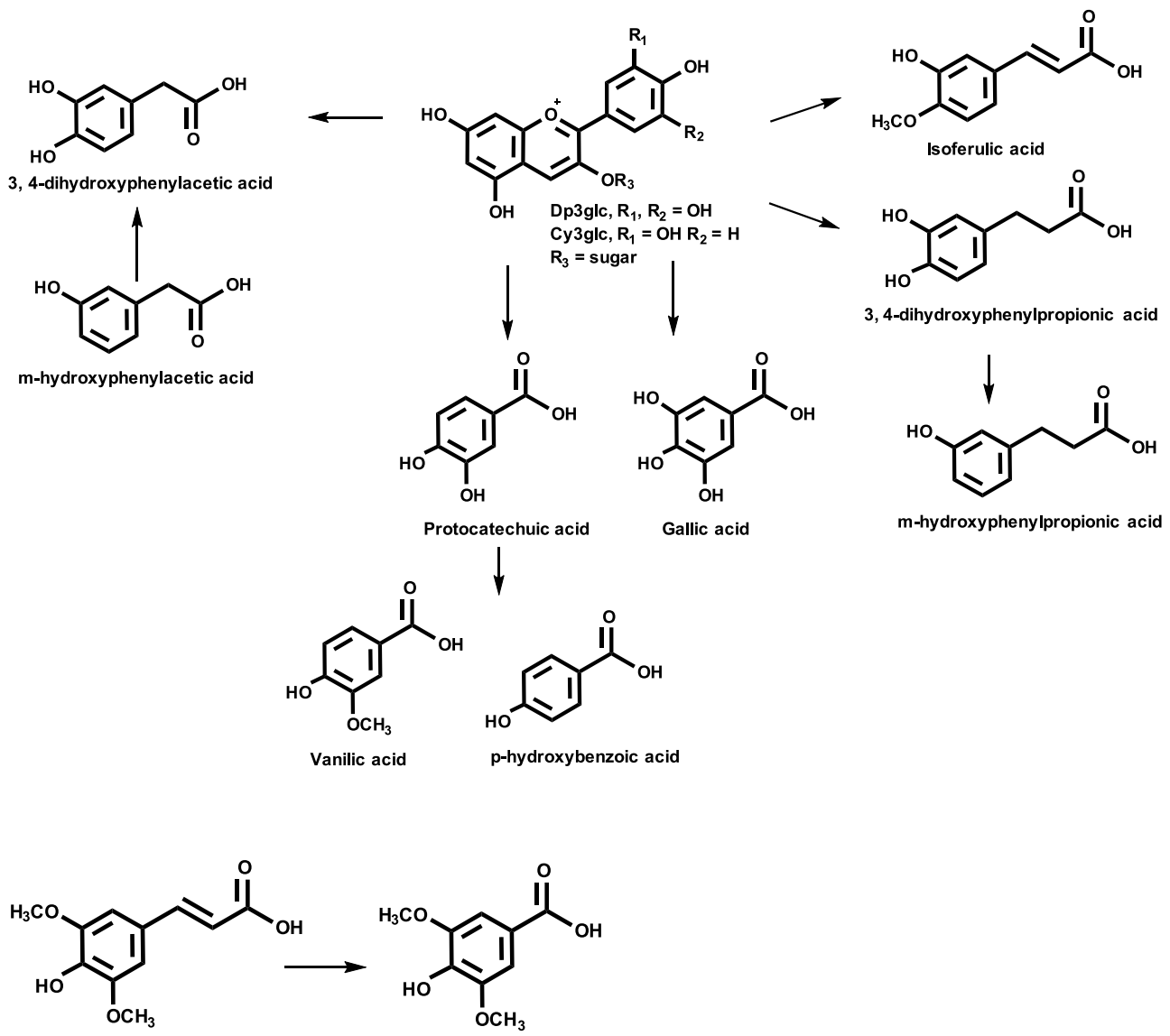

Sinapinic acid

Syringic acid

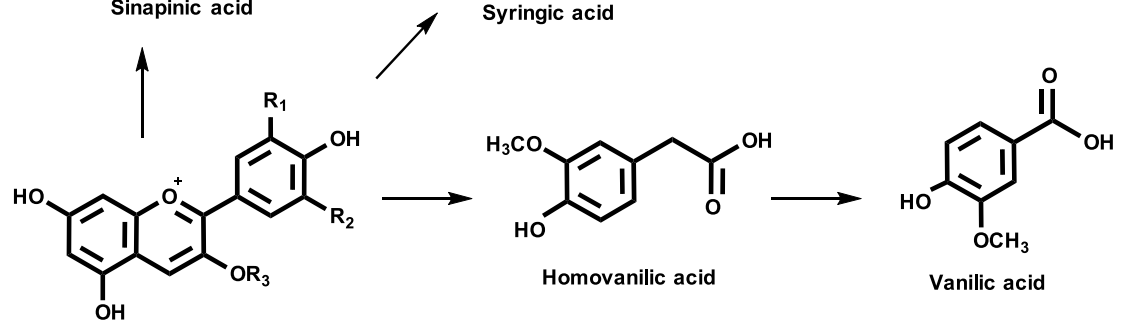

Mv3glc, $\mathrm{R}_{1}, \mathrm{R}_{2}=\mathrm{OCH}_{3}$

Pt3glc, $\mathbf{R}_{\mathbf{1}}=\mathbf{O C H}_{\mathbf{3}} \mathbf{R}_{\mathbf{2}}=\mathrm{OH}$

Pn3glc, $\mathrm{R}_{\mathbf{1}}=\mathrm{OCH}_{3} \mathrm{R}_{\mathbf{2}}=\mathrm{H}$

$\mathbf{R}_{3}=$ sugar

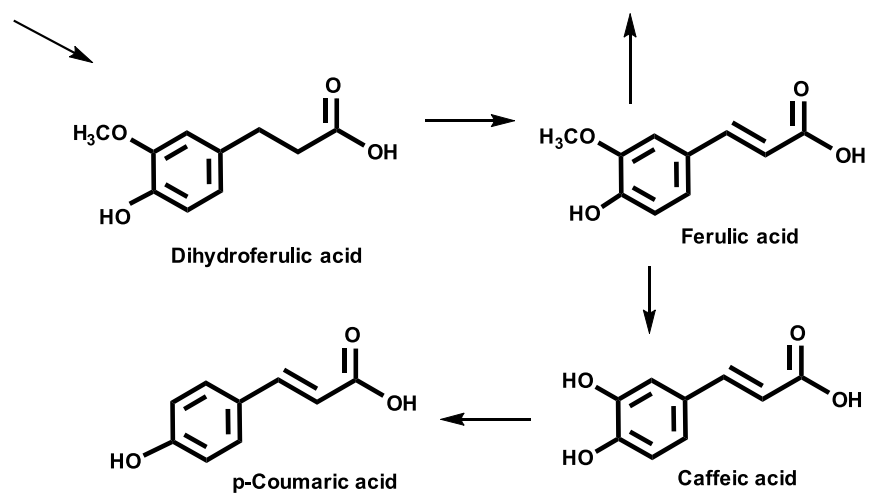

Fig. 5. Catabolites of anthocyanins, adapted from [47]. 
cyanidin-3-glucoside in humans, almost $73 \%$ of the ingested anthocyanins [45]. This metabolite may result from chemical degradation under the physiological conditions of the systemic circulation or in the intestinal mucosa since it was detected in plasma $2 \mathrm{~h}$ after orange juice ingestion. This metabolite is also recovered in fecal samples, which suggests that the gut extensively metabolizes anthocyanins [46]. The metabolism of berry anthocyanins resulting in phenolic acids in humans was recently studied [47]. The main anthocyanin metabolites detected were homovanilic and vanilic acids.

In another study, blueberry anthocyanin absorption and metabolism in rats was accomplished and the main metabolite detected in urine was hippuric acid, which may be produced in liver through a conjugation of glycine with aromatic phenolic acids [48].

Since anthocyanin phenolic acids can be further absorbed in colon [49] it is possible that they are additionally metabolized by hepatic cells [50]. This fact makes the whole field even more complex and opens an additional task to explore, the effect of phase II metabolized catabolites. Furthermore some of these conjugates were already detected, using caco- 2 cell model, namely protocatechuic acid and phloroglucinaldehyde conjugated with glucuronide and sulphate [51].

Bearing all this, health benefits associated with anthocyanin rich foods may also involve the contribution of a continuous release of native or metabolized phenolic compounds into the bloodstream [49]. Since microbial catabolites may be present at many sites of the body in higher concentration than the parent compound, it has been proposed that at least a part of the biological activities ascribed to anthocyanins are due to their colonic catabolites [49].

Despite that, some authors have proved the capacity of intact anthocyanins to reach several tissues/organs. In a recent work, intact anthocyanins were detected in liver, eyes, cortex, and cerebellum of pigs which were fed with diets supplemented with blueberries for 4 weeks [52]. The results suggest that anthocyanins can accumulate in tissues, including tissues beyond the blood-brain barrier. Moreover, two recent works report the capacity of dietary anthocyanins from grapes and berries to reach the brain $[26,53]$.

In a more recent work, proportions of anthocyanin derivatives (methylated anthocyanins and glucuronoconjugated derivatives) were identified in various organs (bladder, prostate, testes, heart and adipose tissue) in rats fed with a blackberry anthocyaninenriched diet [54]. In this study the bladder contained the highest levels of anthocyanins, followed by the prostate. Prostate, testes and heart contained native cyanidin-3-glucoside and a small proportion of cyanidin monoglucuronide. Cyanidin-3-glucoside and methylated derivatives were also present in adipose tissue; this fact seems a little strange considering their hydrophilicity.

Some of the human studies concerning anthocyanin pharmacokinetics were recently reviewed [55]. The overall analysis of the biokinetic parameters has facilitated some main assumptions in what anthocyanin bioavailability is concerned. The most important one is that although there is a considerable variability in the values for the biokinetic parameters, anthocyanins appear to be rapidly absorbed and eliminated, reaching low maximal concentrations in plasma and urine.

\section{Factors affecting anthocyanin bioavailability}

Anthocyanins major route of entry is by oral ingestion, since they are consumed as part of a normal diet. Several factors including variations in the dose, anthocyanin chemical composition in the different sources, food or beverage matrix or processing, age and gender of the individuals can have a huge effect on the bioavailability and metabolism of anthocyanins. In addition, the analytical methodology employed in the studies may indirectly influence the assessment of anthocyanin bioavailability.

The physical-chemical characteristics of anthocyanins, such as the size of the molecule, its water solubility or its $\mathrm{pKa}$ can dictate its ability to cross a membrane. For example, due to their large size, anthocyanins may imply other transport processes rather than diffusion. Moreover, as a result of their hydrophilicity, it is unlikely that anthocyanins could cross membranes freely. Also, anthocyanins may display an ionic charge (positive or negative depending on the tissue) so their absorption may involve other process rather than a simple diffusion. In the acidic gastric environment, the red flavylium cation is the most stable form. However, upon entering the basic environment of the upper small intestine, flavylium cation is most likely changed to carbinol pseudobase which has a limited absorption rate [56].

Anthocyanins can be structurally modified during food processing and storage, and also in vivo 
environments following ingestion. Several studies used in vitro digestive systems to mimic the upper GI tract and determined the potential bioactivity or metabolism of anthocyanins [57, 58]. The GI tract itself can influence the absorption of anthocyanins. Factors such as $\mathrm{pH}$, the presence of food, digestive enzymes, bile acids, microbiota, and the motility and permeability of the GI tract may be determinant in the amount of compound absorbed.

Gastric or intestinal motility and emptying time may influence bioavailability since a higher contact time is usually related with higher absorption.

Considering anthocyanins, food sources, matrices and processing conditions are very important issues that may influence the availability of the compounds. In some cases anthocyanins must be liberated from the food matrix (consumption of fruits), in other examples, the effect of anthocyanin co-ingestion with ethanol, cream and carbohydrates must be considered [59]. Ethanol seems to exert crucial effects on anthocyanins intestinal bioavailability favoring their transport across intestinal epithelia [36]. The ingestion of a non-alcoholic wine resulted in a decrease on anthocyanin bioavailability in comparison with a normal wine [60].

Interestingly, slowing the rate of gastric emptying by co-administering phytic acid [61] or cream [62] increased anthocyanin absorption, again showing that the stomach is a significant site of absorption of these pigments.

Another important factor affecting anthocyanins bioavailability is their possible ingestion as pigments (anthocyanin derivatives), especially when considering wine consumption. Anthocyanin pyruvic-acid adducts can rapidly reach rat plasma after oral administration of malvidin-3-glucoside-pyruvic acid adduct [63]. Also, flavanol-anthocyanin pigments were found to be absorbed in the intestinal caco-2 cell model [64].

Based on the reported studies, a new field of interest that is often overlooked arises: the anthocyanin absorption as anthocyanin-derived pigments, that could act as "pro-anthocyanins" (delivered systems) or could have their own biological impact in the organism.

Therefore, the overall anthocyanin bioavailability should result from the contribution of the amount that crosses all physiological barriers in all their possible forms: native, degradation products, metabolites and anthocyanin derivatives.

There are other additional factors such as age and sex, metabolization, microbiota and protein-binding events that may yield variations in anthocyanin absorption between two different individuals.

Besides the different equilibrium forms depending on the $\mathrm{pH}$ and chemical instability [65], one of the reasons for the complicated picture that has emerged is that many feeds have involved berry or fruit supplements containing several structurally diverse anthocyanins. This makes the complex anthocyanin content of plasma and urine exceedingly difficult, if not impossible, to assess in terms of absorption, excretion and potential phase I and phase II metabolism, especially when $3^{\prime}$-O-methylation can convert cyanidin to peonidin, and delphinidin to petunidin, and $5^{\prime}-\mathrm{O}-$ methylation converts petunidin to malvidin. However, only the first conversion was observed in vitro [66].

These particular features affect anthocyanins absorption, biotransformation, bioavailability, distribution and, as a consequence, their bioactivity.

\section{Gastric absorption of intact anthocyanins}

Until the beginning of the twenty first century few studies had been conducted aiming to understand the fast kinetics of plasma appearance of anthocyanins [22, 24, 35, 67].

In 2003, Passamonti and co-workers performed an in vivo experiment in rats that suggested the possible involvement of the gastric barrier in the rapid detection of anthocyanins in plasma [68]. Additionally, different anthocyanin glycosides were quickly and efficiently absorbed in the stomach (approximately 25\%) and rapidly excreted into bile as intact and metabolized forms [39].

According to the same author, the stomach should not be considered a metabolizing organ since no metabolites were detected [26].

The detection of $p$-hydroxybenzoic acid in stomach $2 \mathrm{~h}$ after ingestion of pelargonidin, may contradicts this assumption [69], although this result is not indicative of anthocyanin transformation in the gastric cavity but rather of the instability and degradation of the anthocyanidin. Similarly, a high proportion (about $20 \%$ ) of red orange anthocyanins was absorbed from the stomach and again no anthocyanin metabolite was observed in the stomach after $30 \mathrm{~min}$ of incubation [70].

The contribution of the gastric mucosa to the metabolization of anthocyanins has not yet been proved, although this tissue possesses conjugative enzyme activities (UDP-glucuronosyltransferase, sulfotrans- 
ferase, and catechol- $O$-methyl transferase) [27-30]. Furthermore, in vitro studies showed that some flavonoids could be metabolized into glucuronidated and sulphated metabolites by the gastric wall $[31,71]$. Therefore, the absence of references on this matter may result from analytical detection problems.

The earliest reference on anthocyanin absorption in stomach is from 2007. In that work, the authors examined the gastric absorption of pelargonidin-3-glucoside using rat models. Once more, a high proportion of pelargonidin-3-glucoside was found to be rapidly absorbed from the stomach (23\%) [72].

All the above data reported the high gastric absorption of anthocyanins and their rapid detection in plasma, although this needs further confirmation with human volunteers.

There are also few references concerning the gastric absorption of other flavonoids. Quercetin, but not quercetin 3-O-glucoside nor quercetin 3-O-rutinoside, was found to be absorbed in rat stomach [73]. Similarly, the isoflavones genistein and daidzein, but not their glucosides, were also found to be absorbed in the rat stomach [71]. On the other hand some phenolic acids were also found to be absorbed at the gastric epithelium [74-77].

The main conclusion common to all the authors is the high content of anthocyanins absorbed in stomach, but the possible mechanism of anthocyanin gastric absorption remains unknown. On this matter, the bioavailability of cyanidin-3-glucoside in rats fed with a red orange by gastric intubation was not significantly affected by simultaneous ingestion of glucose [78]. This fact may suggest that the glucose transporters are not involved in anthocyanin gastric absorption.

A recently published work reported the development of a biologically relevant in vitro model of moderately differentiated adenocarcinoma stomach cells (MKN-28) to be used as a gastric barrier model [79]. This model will allows a first in vitro approach concerning anthocyanin gastric absorption similar to what happens with caco-2 cell line intestinal barrier model. Preliminary studies with this model indicate that the anthocyanins tested did not affected ${ }^{3} \mathrm{H}$-glucose uptake in MKN-28 cell line [80], what may also suggest that glucose transporters are not involved in anthocyanin gastric absorption. Neverthless, a saturable transport for these compounds has been proposed [79]. Other gastric transporter candidates may include glucose transporte 1 (GLUT1), organic anion transporter (OAT2), sodium-coupled monocarboxylate transportes (SMCT1 and SMCT2), since the expression of these transporters has already been detected in stomach tissue [81-83].

A minimal amount of anthocyanins would still be present in the stomach after $4 \mathrm{~h}$, although the interaction of anthocyanins with unidentified proteins in the stomach tissue will reduce their quantitation as free anthocyanins by HPLC [84]. Such binding may be attributed to nonspecific binding or perhaps specific binding to some protein transporter.

The organic anion carrier bilitranslocase is expressed in the stomach $[85,86]$. In vitro, its normal transport activity is competitively inhibited by quinoidal forms of dietary anthocyanins, suggesting that bilitranslocase could promote the facilitated diffusion of anthocyanins at the level of the gastric mucosa [87]. Nevertheless, it should be noted that those in vitro assays were conducted at $\mathrm{pH} 8.0$, which is far from the gastric $\mathrm{pH}$ conditions where no quinoidal forms could be detected (Fig. 3). Therefore, bilitranslocase may be involved in anthocyanin quinoidal forms absorption in the liver or other organs although is not likely to be involved in their gastric absorption [88]. On the other hand, the administration of high amounts of anthocyanins, far from diet levels, could induce saturation of this transport and contribute to the lower anthocyanin bioavailability reported in those particular studies [39]. As it can be easily perceived, there is too much ambiguous information on the literature concerning anthocyanin gastric absorption.

\section{Anthocyanin and/or their metabolites may induce biological activity}

Unlike all other flavonoids, gastric absorption of anthocyanins enables the detection of anthocyanins in their native forms in plasma after few minutes. This feature would not be possible if the intestine was the only absorption barrier, at least in a so high percentage as referred above. The gastric absorption recently described, addresses meaning to the protective effect of native anthocyanins on age related degenerative diseases [89-93]. Before this recently discovery the huge research conducted with native anthocyanins was questionable and had almost no biological significance. Although some works verified that even low plasma concentrations of anthocyanins appear sufficient to induce changes in signal transduction and gene expression in vivo [94, 95]. 


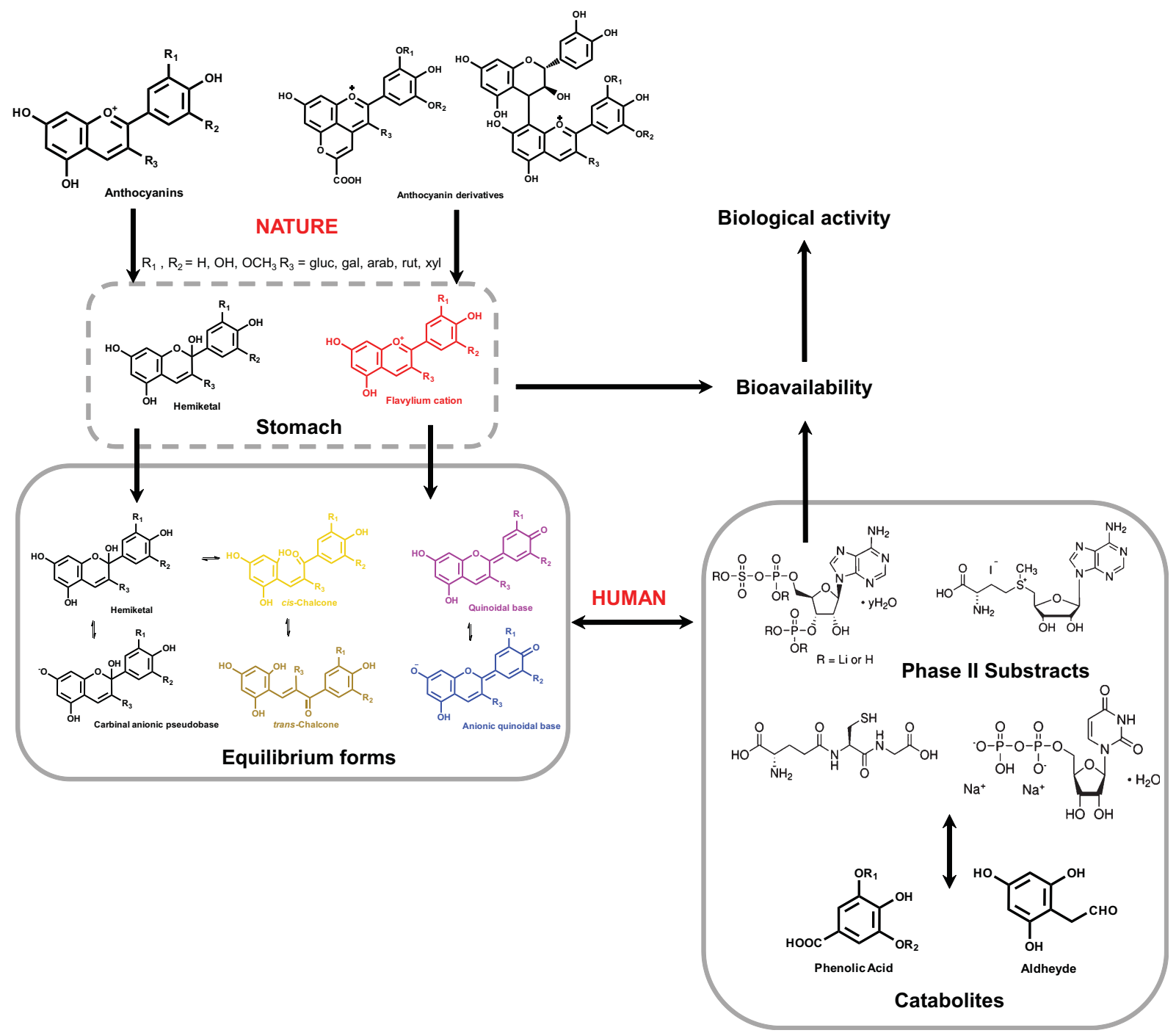

Fig. 6. Schematic representation of the different anthocyanin forms that could contribute to the net biological effects of these food components.

Once absorbed in their flavylium cation or hemiketal forms in the stomach, the native forms that reach the systemic circulation could suffer chemical transformation to all other $\mathrm{pH}$ equilibrium forms and latter be metabolized. The overall anthocyanin bioactivity should result from the contribution of the native structure, degradation products, metabolites and anthocyanin derivatives (Fig. 6).

Considering the several reviews on anthocyanin biological activities, in this topic emphasis will be given to the biological properties of phase II metabolites, phenolic acids and anthocyanin derivatives.

Previously reported in vivo studies with animals and humans have concluded that cyanidin-3-glycoside, delphinidin-3-glycoside and petunidin-3-glycoside are rapidly absorbed and metabolized extensively following oral consumption in humans and rats, to 3'-Me-Cy3glc, 4'-Me-Cy3glc, 4'-Me-Dp3glc and 4'Pt3glc, respectively [96-102].

Based on some of these in vivo studies, an enzymatic approach was conducted and pure standards of methylated anthocyanins were obtained $[66,103]$. The antioxidant and antiproliferative activity against several cancer cell lines (MKN-28, Caco-2 and MCF-7) and the normal human foreskin fibroblasts (HFF1) of these metabolites in comparison with native anthocyanins was evaluated [103]. The methylated metabolites were found to still retain significant radical 
scavenging activity and reducing activity, suggesting that they could act as potential antioxidants, in vivo. The conjugation with methyl groups decreased or did not alter the antiproliferative effect of the original anthocyanin. Neither the metabolites nor the native forms were toxic against the normal human cell line. The achievement of other purified anthocyanin conjugates (glucuronidated and glutathione aducts), not commercially available, will also allow the study of their biological properties [66]. In order to produce higher amounts of metabolites a new chemical synthesis strategy was recently applied and developed to obtain 4'-Me-Cy3glc [104].

Another important feature of anthocyanins is that they have the possibility of being degraded chemically or by the microflora (after enterohepatic recirculation), reaching the blood circulation as cleavage products [105]. Some considerations should also be given to these cleavage products.

Among these catabolites are the phenolic acids that include phenylpropionic, phenylacetic, benzoic acids and m-hydroxyphenylpropionic acids (Fig. 5).

As it can be easily perceived, depending on the anthocyanin chemical structure different phenolic acids may be formed. It should also be highlighted that some of these catabolites could result from the breakdown of other flavonoids or could be ingested as part of the diet. Since the daily intake of anthocyanins has been estimated to be much higher than that of other polyphenols, the nutritional value of phenolic acids derived from their breakdown is increasingly recognized. Moreover, gallic acid is one of the most well-absorbed phenolic compounds [17]. Gallic acid can exert a variety of biological effects by acting on different molecular targets, that include: antioxidant [106], anti-inflammatory [107], antiproliferative activity in different cancer cell lines [108] and may also benefit diabetes patients by triggering the release of insulin by the pancreatic cells [109].

As already referred, protocatechuic acid has been reported as the main metabolite, after anthocyanin consumption [110, 111]. A recent work confirmed the degradation of $\mathrm{Cy} 3 \mathrm{glc}$ to protocatechuic acid after incubation with gut bacteria [112]. Protocatechuic acid possesses antioxidant, anti-inflammatory as well as antihyperglycemic and neuroprotective activities. Furthermore, protocatechuic acid seems to have chemopreventive potential because it inhibits the in vitro chemical carcinogenesis and exerts pro-apoptotic and antiproliferative effects in different tissues [113].
Caffeic and syringic acids have shown to decrease proliferation in leukaemia and human breast cancer cells, whereas no effect was observed in normal cells (HUVEC) [114, 115]. The antiproliferative action on human breast cancer cell growth was also observed for sinapic and ferulic acid [115].

It should be noted that the phase II metabolites may suffer enterohepatic recirculation and can further be degraded and give rise to a new class of phenolic acid that would include the ones methylated in the 4 position and the ones conjugated in the different free $\mathrm{OH}$ groups with sulphate or glucuronyl molecules [49].

Another important contribution to anthocyanin biological activity is their possible absorption as pigments (as anthocyanin derivatives). The antioxidant and antiproliferative activity of anthocyanin-derived pigments is poor evaluated, although there are few references on this topic concerning anthocyanin pyruvic acid adducts and vinylpyranoanthocyanin-catechins [108, 116-118].

\section{Conclusion}

The interest in anthocyanins has been driven primarily by epidemiological studies that have suggested that diets rich in these phytochemicals are beneficial to human health.

As a result of the high gastric absorption, intact anthocyanins or derived pigments could be responsible for a significant proportion of the biological activity derived from consumption of fruits, vegetables and other plant derived products such as fruit drinks and red wine. There is a real possibility that some dietary anthocyanins or derived pigments contribute positively to health and well-being according to the epidemiological studies already performed.

Nevertheless, the biological activity of absorbed parent compounds, their metabolites and microbial catabolites, and the potential synergy between them could be the answer to the anthocyanin paradox bioactivity.

Some questions still lack an accurate answer: what contributes to this huge gap on this field? Why is anthocyanin intestinal absorption always considered the most relevant? Why should colonic metabolites of anthocyanins be the main source of their biological activity? Why is it still not possible to define which transport mechanism is responsible for anthocyanin absorption in stomach? 


\section{Acknowledgments}

This work was funded by the Research Project Grant from the Fundação para a Ciência e Tecnologia (POCI, FEDER, POPH, QREN) (PTDC/AGRTEC/2227/2012) and a Post-PhD grant (SFRH/BPD/ $86173 / 2012)$.

\section{References}

[1] Deroles S, Anthocyanin Biosynthesis in Plant Cell Cultures: A Potential Source of Natural Colourants, in Anthocyanins - Biosynthesis, Functions, and Applications, K. Gould, K. Davies, and C. Winefield, Editors. 2009, Springer. pp. 107169.

[2] Andersen OM, Jordheim M, The anthocyanins, in Flavonoids: Chemistry, biochemistry and applications, A.O. M. and M.K. R., Editors. 2005, CRC Press. pp. 471-552.

[3] Francis FJ. Food colorants: Anthocyanins. Crit Rev Food Sci Nutr. 1989;28(4):273-314.

[4] Mateus N, et al. New Family of Bluish Pyranoanthocyanins. J Biomed Biotechnol. 2004;2004(5):299-305.

[5] Mateus N, et al. Structural diversity of anthocyanin-derived pigments in port wines. Food Chem. 2002;76(3):335-42.

[6] Remy S, et al. First confirmation in red wine of products resulting from direct anthocyanin-tannin reactions. J Sci Food Agric. 2000;80(6):745-51.

[7] Fossen T, Rayyan S, Andersen ØM. Dimeric anthocyanins from strawberry (Fragaria ananassa) consisting of pelargonidin 3-glucoside covalently linked to four flavan-3-ols. Phytochemistry. 2004;65(10):1421-8.

[8] Sentandreu E, Navarro JL, Sendra JM. LC-DAD-ESI/MSn Determination of Direct Condensation Flavanol- Anthocyanin Adducts in Pressure Extracted Pomegranate (Punica granatum L.) Juice. J Agric Food Chem. 2010;58(19):105607.

[9] González-Paramás AM, et al. Flavanol-anthocyanin condensed pigments in plant extracts. Food Chem. 2006; 94(3):428-36.

[10] Brouillard R, Delaporte B. Chemistry of anthocyanin pigments. 2. Kinetic and thermodynamic studt of protontransfer, hydration and tautomeric reactions of malvidin 3 glucoside. Journal of the American Chemical Society. 1977; 99(26):8461-8.

[11] Brouillard R, Dubois JE. Mechanism of structural transformations of anthocyanins in acidic media. J Am Chem Soc. 1977;99(5):1359-64.

[12] Brouillard R, Lang J. The hemiacetal-cis-chalcone equilibrium of malvidin, a natural anthocyanin. Canadian Journal of Chemistry-Revue Canadienne De Chimie. 1990;68(5):75561.

[13] Brouillard R. The in vivo expression of anthocyanin colour in plants. Phytochemistry. 1983;22(6):1311-23.

[14] Mallery SR, et al. Effects of human oral mucosal tissue, saliva and oral microflora on intraoral metabolism and bioactivation of black raspberry anthocyanins. Cancer Prev Res. 2011;4(8):1209-21.
[15] Kamonpatana K, et al. Susceptibility of anthocyanins to ex vivo degradation in human saliva. Food Chem. 2012; 135(2):738-47.

[16] Walle T. Absorption and metabolism of flavonoids. Free Radic Biol Med. 2004;36(7):829-37.

[17] Manach C, et al. Bioavailability and bioefficacy of polyphenols in humans. I. Review of 97 bioavailability studies. Am J Clin Nutr. 2005;81(1 Suppl):230-42.

[18] Vanzo A, et al. Uptake of grape anthocyanins into the rat kidney and the involvement of bilitranslocase. Mol Nutr Food Res. 2008;52(10):1106-16.

[19] Wiese $S$, et al. Protein interactions with cyanidin-3-glucoside and its influence on $\alpha$-amylase activity. J Sci Food Agric. 2009;89(1):33-40.

[20] Matsui T, et al. $\alpha$-Glucosidase Inhibitory Action of Natural Acylated Anthocyanins. 1. Survey of Natural Pigments with Potent Inhibitory Activity. J Agric Food Chem. 2001; 49(4):1948-51.

[21] Walle T, et al. Flavonoid glucosides are hydrolyzed and thus activated in the oral cavity in humans. J Nutr 2005;135(1):4852.

[22] Cao G, Prior RL. Anthocyanins are detected in human plasma after oral administration of an elderberry extract. Clin Chem 1999;45(4):574-6.

[23] Cao G, et al. Anthocyanins are absorbed in glycated forms in elderly women: A pharmacokinetic study. Am J Clin Nutr. 2001;73(5):920-6.

[24] Milbury PE, et al. Bioavailablility of elderberry anthocyanins. Mech Ageing Dev. 2002;123(8):997-1006.

[25] Mulleder U, Murkovic M, Pfannhauser W. Urinary excretion of cyanidin glycosides. J Biochem Biophys Methods. 2002;53(1-3):61-6.

[26] Talavera S, et al. Anthocyanin metabolism in rats and their distribution to digestive area, kidney, and brain. J Agric Food Chem. 2005;53(10):3902-8.

[27] Strassburg CP, et al. Differential Expression of the UGT1A Locus in Human Liver, Biliary, and Gastric Tissue: Identification of UGT1A7 and UGT1A10 Transcripts in Extrahepatic Tissue. Mol Pharmacol. 1997;52(2):212-20.

[28] Strassburg CP, et al. Polymorphic Expression of the UDP-Glucuronosyltransferase UGT1A Gene Locus in Human Gastric Epithelium. Mol Pharmacol. 1998;54(4):64754.

[29] Harris RM, et al. Activity of phenolsulfotransferases in the human gastrointestinal tract. Life Sci. 2000;67(17): 2051-7.

[30] Karhunen T, et al. Distribution of catechol-Omethyltransferase enzyme in rat tissues. J Histochem Cytochem. 1994;42(8):1079-90.

[31] Déchelotte P, et al. Conjugation of 1-naphthol in human gastric epithelial cells. Gut. 1993;34(2):177-80.

[32] Terao J, Murota K, Kawai Y. Conjugated quercetin glucuronides as bioactive metabolites and precursors of aglycone in vivo. Food Funct. 2011;2(1):11-7.

[33] Talavera $S$, et al. Anthocyanins are efficiently absorbed from the small intestine in rats. J Nutr. 2004;134(9):2275-9.

[34] Miyazawa $T$, et al. Direct intestinal absorption of red fruit anthocyanins, cyanidin-3-glucoside and cyanidin-3,5diglucoside, into rats and humans. J Agric Food Chem. 1999; 47(3):1083-91. 
[35] Tsuda T, Horio F, Osawa T. Absorption and metabolism of cyanidin 3-O- $\beta$-D-glucoside in rats. FEBS Lett. 1999;449(23):179-82.

[36] Faria A, et al. Absorption of anthocyanins through intestinal epithelial cells - Putative involvement of GLUT2. Mol Nutr Food Res. 2009;53(11):1430-7.

[37] Walton MC, et al. The flavonol quercetin-3-glucoside inhibits cyanidin-3-glucoside absorption in vitro. J Agric Food Chem. 2006;54(13):4913-20.

[38] Dreiseitel A, et al. Berry anthocyanins and anthocyanidins exhibit distinct affinities for the efflux transporters BCRP and MDR1. Br J Pharmacol. 2009;158(8):1942-50.

[39] Talavera S, et al. Anthocyanins are efficiently absorbed from the stomach in anesthetized rats. J Nutr. 2003;133(12):417882.

[40] McGhie TK, et al. Anthocyanin glycosides from berry fruit are absorbed and excreted unmetabolized by both humans and rats. J Agric Food Chem. 2003;51(16):4539-48.

[41] Ichiyanagi T, et al. Extended glucuronidation is another major path of cyanidin 3-O- $\beta$-D-glucopyranoside metabolism in rats. J Agric Food Chem. 2005;53(18):7312-9.

[42] Ichiyanagi T, et al. Absorption and metabolism of delphinidin 3-O- $\beta$-glucopyranoside in rats. Free Radic Biol Med 2004;36(7):930-7.

[43] Ichiyanagi T, et al. Metabolic pathway of cyanidin 3-O- $\beta$-Dglucopyranoside in rats. J Agric Food Chem. 2005;53(1):14550 .

[44] Woodward G, et al. Anthocyanin Stability and Recovery: Implications for the Analysis of Clinical and Experimental Samples. J Agric Food Chem 2009;57(12):5271-8.

[45] Vitaglione P, et al. Protocatechuic acid is the major human metabolite of cyanidin-glucosides. J Nutr. 2007;137(9):20438.

[46] Riso P, et al. Effects of blood orange juice intake on antioxidant bioavailability and on different markers related to oxidative stress. J Agric Food Chem. 2005;53(4):941-7.

[47] Nurmi T, et al. Metabolism of Berry Anthocyanins to Phenolic Acids in Humans. J Agric Food Chem. 2009;57(6): 2274-81.

[48] Del Bò C, et al. Anthocyanin Absorption, Metabolism, and Distribution from a Wild Blueberry-Enriched Diet (Vaccinium angustifolium) Is Affected by Diet Duration in the Sprague-Dawley Rat. J Agric Food Chem. 2009;58(4):24917.

[49] Williamson G, Clifford MN. Colonic metabolites of berry polyphenols: The missing link to biological activity? $\mathrm{Br} \mathrm{J}$ Nutr. 2010;104:S48-66.

[50] Woodward GM, Needs PW, Kay CD. Anthocyanin-derived phenolic acids form glucuronides following simulated gastrointestinal digestion and microsomal glucuronidation. Mol Nutr Food Res. 2011;55(3):378-86.

[51] Kay CD, Kroon PA, Cassidy A. The bioactivity of dietary anthocyanins is likely to be mediated by their degradation products. Mol Nutr Food Res. 2009;53(S1):92-101.

[52] Kalt W, et al. Identification of Anthocyanins in the Liver, Eye, and Brain of Blueberry-Fed Pigs. J Agric Food Chem. 2008;56(3):705-12.

[53] Passamonti S, et al. Fast Access of Some Grape Pigments to the Brain. J Agric Food Chem. 2005;53(18):7029-34.
[54] Felgines C, et al. Tissue distribution of anthocyanins in rats fed a blackberry anthocyanin-enriched diet. Mol Nutr Food Res. 2009;53(9):1098-103.

[55] Faria A, et al., Bioavailability of anthocyanins, in Handbook of Natural Products, R. K. and J.-M. Merillon, Editors. 2013, Springer: Germany.

[56] Crozier A, Jaganath IB, Clifford MN. Dietary Phenolics: Chemistry, Bioavailability and Effects on Health. Nat Prod Rep. 2009;40(46).

[57] McDougall GJ, et al. Assessing Potential Bioavailability of Raspberry Anthocyanins Using an in Vitro Digestion System. J Agric Food Chem. 2005;53(15):5896-904.

[58] McDougall GJ, et al. Anthocyanins from red wine - Their stability under simulated gastrointestinal digestion. Phytochemistry. 2005;66(21):2540-8.

[59] Yang M, et al. Food matrix affecting anthocyanin bioavailability: Review. Curr Med Chem. 2011;18(2):291-300.

[60] Scholz S, Williamson G. Interactions affecting the bioavailability of dietary polyphenols in vivo. Int J Vitam Nutr Res. 2007;77(3):224-35.

[61] Matsumoto H, et al. Enhanced absorption of anthocyanins after oral administration of phytic acid in rats and humans. J Agric Food Chem. 2007;55(6):2489-96.

[62] Mullen W, et al. Bioavailability of Pelargonidin-3-Oglucoside and Its Metabolites in Humans Following the Ingestion of Strawberries with and without Cream. J Agric Food Chem. 2008;56(3):713-9.

[63] Faria A, et al. Bioavailability of anthocyanin-pyruvic acid adducts in rat. in International Conference on Polyphenols and Health. 2009. Yorkshire, Leeds.

[64] Fernandes I, et al. On the bioavailability of flavanols and anthocyanins: Flavanol-anthocyanin dimers. Food Chem. 2012;135(2):812-8

[65] McGhie TK, Walton MC. The bioavailability and absorption of anthocyanins: Towards a better understanding. Mol Nutr Food Res. 2007;51(6):702-13.

[66] Fernandes I, et al. Enzymatic hemisynthesis of metabolites and conjugates of anthocyanins. J Agric Food Chem. 2009;57(2):735-45.

[67] Murkovic M, et al. Detection of anthocyanins from elderberry juice in human urine. J Sci Food Agric. 2001;81(9): 934-7.

[68] Passamonti S, et al. The stomach as a site for anthocyanins absorption from food. FEBS Lett. 2003;544(1-3):210-3.

[69] El Mohsen MA, et al. Absorption, tissue distribution and excretion of pelargonidin and its metabolites following oral administration to rats. Br J Nutr. 2006;95(01):51-8.

[70] Felgines $\mathrm{C}$, et al. Absorption and metabolism of red orange juice anthocyanins in rats. Br J Nutr. 2006;95(5):898-904.

[71] Piskula MK, Yamakoshi J, Iwai Y. Daidzein and genistein but not their glucosides are absorbed from the rat stomach. FEBS Lett. 1999;447(2):287-91.

[72] Felgines C, et al. Strawberry pelargonidin glycosides are excreted in urine as intact glycosides and glucuronidated pelargonidin derivatives in rats. Br J Nutr. 2007;98(6):112631.

[73] Crespy V, et al. Quercetin, but not its glycosides, is absorbed from the rat stomach. J Agric Food Chem. 2001;50(3):61821. 
[74] Konishi Y, Zhao Z, Shimizu M. Phenolic acids are absorbed from the rat stomach with different absorption rates. J Agric Food Chem. 2006;54(20):7539-43.

[75] Lafay S, et al. Chlorogenic acid is absorbed in its intact form in the stomach of rats. J Nutr. 2006;136(5):1192-7.

[76] Vanzo A, et al. The Fate of trans-Caftaric acid administered into the rat stomach. J Agric Food Chem. 2007;55(4):160411.

[77] Zhao Z, Egashira Y, Sanada H. Ferulic acid is quickly absorbed from rat stomach as the free form and then conjugated mainly in liver. J Nutr. 2004;134(11):3083-8.

[78] Felgines C, et al. Influence of glucose on cyanidin 3-glucoside absorption in rats. Mol Nutr Food Res. 2008;52(8):959-64.

[79] Fernandes I, et al. A new approach on the gastric absorption of anthocyanins. Food Funct. 2012;3(5):508-16.

[80] Fernandes I, et al. Bioavailability of anthocyanins and derivatives. Journal of Functional Foods. 2013.

[81] Yoshikawa T, et al. Comparative expression of hexose transporters (SGLT1, GLUT1, GLUT2 and GLUT5) throughout the mouse gastrointestinal tract. Histochemistry and Cell Biology. 2011;135(2):183-94.

[82] Eraly SA, et al. The Molecular pharmacology of organic anion transporters: from DNA to FDA? mol mharmacol. 2004;65(3):479-87.

[83] Garcia CK, et al. cDNA cloning of MCT2, a second monocarboxylate transporter expressed in different cells than MCT1. Journal of Biological Chemistry. 1995;270(4):1843-9.

[84] He J, et al. Stability of black raspberry anthocyanins in the digestive tract lumen and transport efficiency into gastric and small intestinal tissues in the rat. J Agric Food Chem. 2009;57(8):3141-8

[85] Battiston L, et al. Specific sequence-directed antibilitranslocase antibodies as a tool to detect potentially bilirubin-binding proteins in different tissues of the rat. FEBS Lett. 1999;453(3):351-5.

[86] Nicolin V, et al. Immunolocalisation of bilitranslocase in mucosecretory and parietal cells of the rat gastric mucosa. Journal of Molecular Histology. 2005;36(1):45-50.

[87] Passamonti S, Vrhovsek U, Mattivi F. The interaction of anthocyanins with bilitranslocase. Biochem Biophys Res Commun. 2002;296(3):631-6.

[88] Passamonti S, et al. Hepatic uptake of grape anthocyanins and the role of bilitranslocase. Food Chem Toxicol. 2005;38(89):953-60.

[89] Shukitt-Hale B, Lau FC, Joseph JA. Berry Fruit Supplementation and the Aging Brain. J Agric Food Chem. 2008; 56(3):636-41.

[90] Andres-Lacueva C, et al. Anthocyanins in aged blueberryfed rats are found centrally and may enhance memory. Nutr Neurosci. 2005;8(2):111-20.

[91] Kang TH, et al. Neuroprotective effects of the cyanidin-3$\mathrm{O}-\beta$-d-glucopyranoside isolated from mulberry fruit against cerebral ischemia. Neuroscience Letters. 2006;391(3):122-6.

[92] Lazze MC, et al. Anthocyanins induce cell cycle perturbations and apoptosis in different human cell lines. Carcinogenesis. 2004;25(8):1427-33.

[93] Mazza G, et al. Absorption of anthocyanins from blueberries and serum antioxidant status in human subjects. J Agric Food Chem. 2002;50(26):7731-7.
[94] Karlsen A, et al. Anthocyanins inhibit nuclear factor-kappaB activation in monocytes and reduce plasma concentrations of pro-inflammatory mediators in healthy adults. J Nutr. 2007;137(8):1951-4.

[95] DeFuria J, et al. Dietary blueberry attenuates whole-body insulin resistance in high fat-fed mice by reducing adipocyte death and its inflammatory sequelae. J Nutr. 2009;139(8): 1510-6.

[96] Felgines C, et al. Blackberry anthocyanins are mainly recovered from urine as methylated and glucuronidated conjugates in humans. J Agric Food Chem. 2005;53(20):7721-7.

[97] Kay CD, et al. Anthocyanin metabolites in human urine and serum. Br J Nutr. 2004;91(6):933-42.

[98] Kay CD, Mazza GJ, Holub BJ. Anthocyanins exist in the circulation primarily as metabolites in adult men. $\mathbf{J}$ Nutr. 2005;135(11):2582-8.

[99] Tian Q, et al. Urinary excretion of black raspberry (Rubus occidentalis) anthocyanins and their metabolites. J Agric Food Chem. 2006;54(4):1467-72.

[100] Ichiyanagi T, et al. Effect on both aglycone and sugar moiety towards Phase II metabolism of anthocyanins. Food Chem. 2008;110(2):493-500.

[101] Marczylo T, et al. Pharmacokinetics and metabolism of the putative cancer chemopreventive agent cyanidin-3-glucoside in mice. Cancer Chemother Pharmacol. 2009;64(6):1261-8.

[102] Ichiyanagi T, et al. Bioavailability and tissue distribution of anthocyanins in bilberry (Vaccinium myrtillus $L$.) extract in rats. J Agric Food Chem. 2006;54(18):6578-87.

[103] Fernandes I, et al. Antioxidant and antiproliferative properties of methylated metabolites of anthocyanins. Food Chem. 2013;141(3):2923-33.

[104] Cruz L, Mateus N, de Freitas V. First chemical synthesis report of an anthocyanin metabolite with in vivo occurrence: Cyanidin-4'-O-methyl-3-glucoside. Tetrahedron Lett. 2013;54(22):2865-9.

[105] Gonthier M-P, et al. Microbial aromatic acid metabolites formed in the gut account for a major fraction of the polyphenols excreted in urine of rats fed red wine polyphenols. J Nutr. 2003;133(2):461-7.

[106] Rasool MK, et al. Hepatoprotective and antioxidant effects of gallic acid in paracetamol-induced liver damage in mice. Journal of Pharmacy and Pharmacology. 2010;62(5): 638-43.

[107] Kim S-H, et al. Gallic acid inhibits histamine release and pro-inflammatory cytokine production in mast cells. Toxicological Sciences. 2006;91(1):123-31.

[108] Fernandes I, et al. Influence of anthocyanins, derivative pigments and other catechol and pyrogallol-type phenolics on breast cancer cell proliferation. J Agric Food Chem. 2010;58(6):3785-92.

[109] Sameermahmood Z, et al. Gallic acid protects RINm5F $\beta$ cells from glucolipotoxicity by its antiapoptotic and insulinsecretagogue actions. Phytother Res. 2010;24(S1):S83-94.

[110] Goldberg DM, Yan J, Soleas GJ. Absorption of three winerelated polyphenols in three different matrices by healthy subjects. Clin Biochem. 2003;36(1):79-87.

[111] Selma MV, Espin JC, Tomas-Barberan FA. Interaction between phenolics and gut microbiota: Role in human health. J Agric Food Chem. 2009;57(15):6485-501. 
[112] Hanske L, et al. Contribution of gut bacteria to the metabolism of cyanidin 3-glucoside in human microbiota-associated rats. Br J Nutr. 2013;109(8):1433-41.

[113] Masella R, et al. Protocatechuic acid and human disease prevention: biological activities and molecular mechanisms. Curr Med Chem. 2012;19(18):2901-17.

[114] Zambonin L, et al. Dietary phenolic acids act as affective antioxidants in membrane models and in cultured cells, exhibiting proapoptotic effects in leukaemia cells. Oxid Med Cell Longev. 2012;2012:12.

[115] Kampa M, et al. Antiproliferative and apoptotic effects of selective phenolic acids on T47D human breast cancer cells: Potential mechanisms of action. Breast Cancer Res. 2004;6(2):R63-74.
[116] Faria A, et al. Blueberry anthocyanins and pyruvic acid adducts: Anticancer properties in breast cancer cell lines. Phytother Res. 2010;24(12):1862-9.

[117] Faria A, et al. Antioxidant properties of prepared blueberry (Vaccinium myrtillus) extracts. J Agric Food Chem. 2005;53(17):6896-902.

[118] Azevedo J, et al. Antioxidant properties of anthocyanidins, anthocyanidin-3-glucosides and respective portisins. Food Chem. 2010;119(2):518-23.

[119] Nave F, et al. Thermodynamic and kinetic properties of a red wine pigment: Catechin-(4,8)-malvidin-3-O-glucoside. J Phys Chem B. 2010;114(42):13487-96. 